\title{
Clinical Assessment of Alzheimer's Disease and Response to Donepezil Treatment
}

\author{
S. Gauthier*, M.D., F.R.C.P.(C).
}

\section{THE CASE}

Mr. AB was assessed at the McGill Centre for Studies in Aging in December 1995 at the age of 73 for a two year history of decreased recall for recent events and hesitation for words. University educated and active socially, living with his spouse there was some interference of these symptoms with daily life: he had to be reminded of appointments and used the expression "this thing" more and more in his spontaneous language. His medical background was unremarkable and an older brother was a little forgetful.

The neurologic assessment showed a slight difficulty to draw a clock with instructions to put all the numbers and set the time at 11:10. He had no observable impairment of copying a cube The score on the Mini Mental State Examination (MMSE) (1) was $27 / 30$, well into the normal range.

Uninfused brain computer tomography (CT) imaging showed moderate diffuse brain atrophy evident by widening of the convolutional sulci and mild dilatation of the lateral ventricles.

A diagnosis of dementia caused by Alzheimer's disease (AD) was suspected but follow-up assessments were required for confirmation: six months later his family reported that he repeated questions often, with more hesitation for words leading to angry verbal outbursts; the MMSE score was the same. Nine months after the initial visit the MMSE score dropped to $24 / 30$ and the repeat CT showed increasing atrophy.

A diagnosis of $\mathrm{AD}$ was made, and explained to the patient and his family. He was offered to join a

\footnotetext{
* To whom correspondence should be addressed: Alzheimer's Disease Research Unit, McGill Centre for Studies in Aging, 6825 LaSalle Blvd, Verdun, Québec, Canada, H4H 1R3
}

research protocol evaluating the safety and efficacy of Cerebrolysine, a mixture of peptides extracted from pig brain and having demonstrated in vitro neurotropic properties. Administered intravenously, the treatment allocation was randomized to placebo or active infusion once a day for 28 days at home. After five months of follow-up, there was no detectable change in clinical status and the MMSE score was 23/30.

The patient elected to start donepezil (Aricept) therapy, a cholinesterase inhibitor acting by enhancing residual acetylcholine activity. Prior to treatment hobbies and interest were listed, including reading of newspapers intermittently and having stopped more complex tasks such as crossword puzzles and playing chess.

After one month on donepezil $5 \mathrm{mg}$ once a day, he described an improved recall for upcoming events, had played and won a chess game against his son (and remembered winning it), and his MMSE score had increased to 28/30. After two months of treatment, there was an apparent loss of clinical benefit, and the MMSE score was 24/30. The dose of donepezil was increased to $10 \mathrm{mg}$ daily. After four months the family reported a decreased in the number of questions repeated, and the MMSE was 25/30. After six months, he is stable clinically. At no time did gastro-intestinal side-effects such as nausea, diarrhea or vomiting occur.

\section{DIFFERENTIAL DIAGNOSIS AND WORK-UP}

The diagnosis of dementia requires a decline in memory and at least one other cognitive domain, such as language, interfering with daily life, and representing a decline from a previous level of performance, ruling out delirium and primary psychiatric disorders such as 
major depression (2). Furthermore, the presentation and early course should fit a "typical" profile, i.e., progressive cognitive impairment with no behavioral changes and no motor signs (3).

In the case of highly intelligent and educated individuals, the MMSE may be falsely normal and the clock drawing test (4) is a useful adjunct.

The Canadian Consensus Conference on the Assessment of Dementia has recommended a minimum of laboratory work to be performed in patients with typical $\mathrm{AD}$, looking for concomitant and reversible conditions: CBC, serum electrolytes, TSH, glycemia (5).

Anatomical brain imaging using $\mathrm{CT}$ usually adds little to the diagnosis of typical AD (6). Magnetic resonance imaging scanning could be useful if a vascular component was suspected based on history of abrupt onset, step-wise progression, history of arterial hypertension or stroke, and lateralized neurological symptoms and signs. Single photon emission tomography may prove useful if a frontal lobe dementia is suspected, demonstrating frontal hypoperfusion. Positron emission tomography is not routinely performed, but would have demonstrated hypometabolism in the associative cortices of the temporal and parietal lobes.

\section{MANAGEMENT}

Once the clinical diagnosis of $\mathrm{AD}$ has been well established by history, objective mental status assessment, serial observations demonstrating deterioration, and ruling out or treating concomitant illnesses such as depression, it is the physician's responsibility to inform the patient and advise regarding will and advance directives, monitor driving abilities, and discuss realistically the potential advantages of symptomatic drugs available under prescription and others under testing (7).

\section{DISCUSSION}

This patient was fortunate to be able to participate in a randomized therapeutic study assessing the safety and efficacy of a neurotropic agent. Still under the blind we do not know if he has been on active treatment or placebo. A recent report has demonstrated that participation in clinical trials, whether on placebo or active drug therapy, has a long term benefit (8). It is the opinion of the AD Society of Canada that patients with $\mathrm{AD}$ have the right participate in research (9), with safeguards as to the level of potential benefit and risk at different stages of the disease (10).

The patient then decided to try an established symptomatic drug therapy using donepezil. His therapeutic response was rapid and dramatic, as reflected by the 5 points improvement on the MMSE score. There was a small, but important to the patient and family, improvement in performance of complex hobbies. He remains autonomous for instrumental and self-care activities after six months of therapy with donepezil, justifying a continuation of treatment beyond the six months duration of pivotal randomized placebo-controlled studies (11). Furthermore, observations of patients on continuous therapy with donepezil demonstrate a stable benefit on cognition up to 38 weeks (12).

It is not known if a structured approach to cognitive training would amplify the effects of pharmacotherapy in $\mathrm{AD}$. Combination of drugs acting on cholinergic synaptic activity could also be of interest, paying attention to additive cholinergic side-effects. Finally, combination of donepezil with agents having a potential stabilization effect on disease progression are logical but require further study (13).

\section{REFERENCES}

1. Folstein MF, Folstein SE, McHugh PR. Mini Mental State: a practical method for grading the cognitive state of patients for the clinician. Journal of Psychiatric Research 12(3): 189$198 ; 1975$.

2. American Psychiatric Association. Diagnostic and Statistical Manual of Mental Disorders, 4th edition. Washington DC: American Psychiatric Association; 1994.

3. Gauthier S, Panisset M, Nalbantoglu J, Poirier J. Alzheimer's disease: current knowledge, management andcresearch. Canadian Medical Association Journal 157(8): 1047-1052; 1997.

4. Thalman B, Mansch AU, Ermini-Fiinfshilling D et al. Improved screening for dementia: combining the Clock Drawing Test and the Mini-Mental Status Examination. Presented at the 4th International Nice/Springfield Alzheimer Symposium, Nice, April 10-14th 1996.

5. Organizing Comittee, Canadian Consensus Confgerence on the Assessment of Dementia. Canadian Medical Association Journal 144: 851-853; 1991.

6. Fontaine S, Nordberg A. Brain imaging. In: Clinical Diagnosis and Management of Alzheimer's Disease. Gauthier S, ed., London: Martin Dunitz Ltd; 1996: 83-106.

7. Gauthier S, Burns A, Pettit W. Alzheimer's Disease in Primary Care. London: Martin Dunitz Ltd;1997.

8. Albert SM, Sano M, Marder K, et al. Participation in clinical trials and long-term outcomes ijn Alzheimer's disease. Neurology 49(1): 38-43; 1997.

9. Communicating the diagnosis. In: Tough Issues. Alzheimer Society of Canada; 1997.

10. Keyserlingk EW, Glass K, Kogan S, Gauthier S. Proposed guidelines for the participation of persons with dementia as research subjects. Perspectives in Biology and Medicine 38: 319-362; 1995.

11. Rogers SL, Farlow MR, Doody RS et al. A 24-week, doubleblind, placebo-controlled trial of donepezil in patients with Alzheimer's disease. Neurology 50: 136-145; 1998.

12. Rogers SL, Friedhoff LT. Long-term efficacy and safety of donepezil in the treatment of Alzheimer's disease: an interim analysis of the results of a US multicentre open label 
extension study. European Neuropsychopharmacology 8: 67$75 ; 1998$
13. Gauthier S. Treatment strategies for Alzheimer's disease. McGill Journal of Medicine 3(2): 149-152; 1998

Serge Gauthier, M.D., is a neurologist with special interest in the treatment of Alzheimer's disease. After his M.D. degree at the Université de Montréal (Montreal, Quebec, Canada), he completed his Neurology training at McGill University (Montreal, Quebec, Canada) followed by a MRC Scholarship with Professor Theodore L, Sourkes. After ten years as Director of the McGill Centre for Studies in Aging (Montreal, Quebec, Canada), he is currently a recipient of a MRC/PMAC Senior Scientist Award. 